\title{
Entanglement renormalization for weakly interacting fields
}

\author{
Jordan S. Cotler, ${ }^{1, *}$ M. Reza Mohammadi Mozaffar, ${ }^{2, \dagger}$ Ali Mollabashi, ${ }^{2,}$ and Ali Naseh ${ }^{3, \S}$ \\ ${ }^{1}$ Stanford Institute for Theoretical Physics, Stanford University, Stanford, California 94305, USA \\ ${ }^{2}$ School of Physics, Institute for Research in Fundamental Sciences (IPM), \\ P.O. Box 19395-5531, Tehran, Iran \\ ${ }^{3}$ School of Particles and Accelerators, Institute for Research in Fundamental Sciences (IPM), \\ P.O. Box 19395-5531, Tehran, Iran
}

(Received 4 September 2018; published 16 April 2019)

\begin{abstract}
We adapt the techniques of entanglement renormalization tensor networks to weakly interacting quantum field theories in the continuum. A key tool is "quantum circuit perturbation theory," which enables us to systematically construct unitaries that map between wave functionals which are Gaussian with arbitrary perturbative corrections. As an application, we construct a local continuous multiscale entanglement renormalization ansatz (cMERA) circuit that maps an unentangled scale-invariant state to the ground state of $\varphi^{4}$ theory to one loop. Our local cMERA circuit corresponds exactly to one-loop Wilsonian renormalization group (RG) flow on the spatial momentum modes. In other words, we establish that perturbative Wilsonian RG on spatial momentum modes can be equivalently recast as a local cMERA circuit in $\varphi^{4}$ theory and argue that this correspondence holds more generally. Our analysis also suggests useful numerical ansätze for cMERA in the nonperturbative regime.
\end{abstract}

DOI: 10.1103/PhysRevD.99.085005

\section{INTRODUCTION}

Tensor networks have become a transformative tool for numerically analyzing one-dimensional (1D) quantum systems on the lattice, as well as exploring properties of 1D many-body states with area-law entanglement [1-9]. However, there are various obstructions to generalizing tensor networks (i) to higher dimensions and (ii) to continuum field theories. Tensor networks for higherdimensional lattices are computationally difficult to implement since accurate numerics can require an intractably large number of tensor contractions, even for systems of modest size [10]. On the other hand, the predominant continuum tensor network cMPS (continuous matrix product states) is useful for nonrelativistic systems in $1+1$ dimensions, but fails for relativistic systems in $1+1$ dimensions and also suffers from the "contraction problem" in higher dimensions [11-14].

A promising tensor network architecture designed to work both in higher dimensions and in the continuum is

\footnotetext{
j.jcotler@stanford.edu

m_mohammadi@ipm.ir

\#mollabashi@ipm.ir

§naseh@ipm.ir
}

Published by the American Physical Society under the terms of the Creative Commons Attribution 4.0 International license. Further distribution of this work must maintain attribution to the author(s) and the published article's title, journal citation, and DOI. Funded by SCOAP ${ }^{3}$. called cMERA, the continuum analog of MERA (multiscale entanglement renormalization ansatz) [15]. cMERA generates variational ansätze which have a hierarchical pattern of entanglement across distance scales. The construction of the cMERA state is inspired by spatial renormalization group ( $\mathrm{RG})$ methods. Each layer of the network composing the state corresponds to a step of renormalization group flow.

For all of its promise, cMERA has only been constructed for the ground states of free field theories which are solvable using standard methods [15-18]. Even in the context of mean field theory, cMERA has limited utility over known methods [19].

In this paper, we take the first steps towards applying cMERA to interacting field theories. In particular, we use quantum circuit perturbation theory to construct a cMERA for the ground state of $\varphi^{4}$ theory to one loop in perturbation theory. Remarkably, we can construct a spatially local cMERA circuit which corresponds exactly to one-loop Wilsonian RG on spatial momentum modes. We argue that this equivalence between Wilsonian RG and spatially local tensor networks holds more generally. This equivalence addresses a crucial conceptual and technical gap between momentum space and position space RG methods, whose precise relationship was previously not known. Further, our analysis provides a bridge between the analytic Wilsonian techniques used in high energy physics for momentum space $\mathrm{RG}$ and the algebraic tensor network techniques introduced by quantum information theorists for real space RG. 
Our perturbative analysis of cMERA suggests numerical cMERA ansätze for ground states of interacting theories, which need not have weak coupling. We explore a numerical ansatz in the Appendix.

\section{CIRCUIT PERTURBATION THEORY}

Before constructing cMERA circuits for weakly interacting field theories, we need to gain facility with manipulating quantum circuits for quantum field theories (QFTs). Our core tool is quantum circuit perturbation theory, which we summarize here. For a detailed user guide, see [20]. A central question is, given two states of a quantum field theory $\left|\Psi_{1}\right\rangle$ and $\left|\Psi_{2}\right\rangle$ which are each Gaussian with arbitrary perturbative corrections, how do we systematically construct a unitary $U$ such that $U\left|\Psi_{1}\right\rangle=\left|\Psi_{2}\right\rangle$ ?

For concreteness, we restrict our attention to scalar field theory in $d$ dimensions. The canonical commutation relations are $[\hat{\phi}(\vec{x}), \hat{\pi}(\vec{y})]=i \delta^{d}(\vec{x}-\vec{y})$. A Gaussian state $|\Psi\rangle$ has a wave functional of the form

$$
\langle\phi \mid \Psi\rangle=\mathcal{N} e^{-\frac{1}{2} \int d^{d} \vec{x} d^{d} \vec{y}(\phi(\vec{x})-a(\vec{x})) b(\vec{x}, \vec{y})(\phi(\vec{y})-a(\vec{y}))},
$$

where $\mathcal{N}$ is an overall normalization. Throughout the paper, we will use $\mathcal{N}$ as a placeholder for normalization. We see that the state is completely determined by its one- and twopoint correlation functions. We will be primarily interested in translation- and rotation-invariant Gaussian states-this corresponds to states of the form in Eq. (1) for which $a(\vec{x})=$ const and $b(\vec{x}, \vec{y})=b(|\vec{x}-\vec{y}|)$.

In scalar field theory, we can write any nonsingular Hermitian operator $O$ as

$O=\sum_{n=0}^{\infty} \sum_{k=0}^{n} \int d^{d} \vec{x}_{1} \cdots d^{d} \vec{x}_{n} c_{n}^{(k)}\left(\vec{x}_{1}, \ldots, \vec{x}_{n}\right) S_{n}^{(k)}\left(\vec{x}_{1}, \ldots, \vec{x}_{n}\right)$,

where all of the $c_{n}^{(k)}$ are real-valued functions or distributions, and

$S_{n}^{(k)}\left(\vec{x}_{1}, \ldots, \vec{x}_{n}\right)=\hat{\phi}\left(\vec{x}_{1}\right) \cdots \hat{\phi}\left(\vec{x}_{k}\right) \hat{\pi}\left(\vec{x}_{k+1}\right) \cdots \hat{\pi}\left(\vec{x}_{n}\right)+$ H.c.,

where $1 \leq k \leq n$. In other words, $\left\{S_{n}^{(k)}\right\}$ generates all operators in the theory. The quadratic operators, which are generated by $\left\{S_{n}^{(k)}\right\}_{n \leq 2}$, have particularly nice properties: they form a (closed) Lie algebra and generate unitaries which map Gaussian states to Gaussian states. In terms of equations, if $Q, Q^{\prime}$ are quadratic operators, then $\left[Q, Q^{\prime}\right]$ is also a quadratic operator. If $|\Psi\rangle$ is a Gaussian state, then $e^{-i Q}|\Psi\rangle$ is also a Gaussian state.

Given two (translation- and rotation-invariant) Gaussian states $\left|\Psi_{1}^{G}\right\rangle,\left|\Psi_{2}^{G}\right\rangle$, one can systematically find quadratic operators $Q$ such that $e^{-i Q}\left|\Psi_{1}^{G}\right\rangle=\left|\Psi_{2}^{G}\right\rangle$. This systematic construction is possible due to the technology of squeezed coherent states, which leverages that quadratic operators form a manageably small (closed) Lie algebra. By contrast, given two non-Gaussian states, it is generally not possible to systematically construct unitaries which map between the states. This problem amounts to considering the equation $e^{-i O}\left|\Psi_{1}\right\rangle=\left|\Psi_{2}\right\rangle$ for a generic $O$ (as per Eq. (2)) with undetermined $c_{n}^{(k)}$,s, and then finding $c_{n}^{(k)}$,s which satisfy the equation.

Luckily, there is a tractable middle ground between the Gaussian and non-Gaussian cases. Suppose we have some small parameter $\epsilon$, and that $\left|\Psi_{1}\right\rangle,\left|\Psi_{2}\right\rangle$ are Gaussian up to perturbative corrections in $\epsilon$. Specifically, suppose that we consider first order corrections in $\epsilon$ of the form

$\left|\Psi_{1}\right\rangle=\left(1-i \epsilon R_{1}\right)\left|\Psi_{1}^{G}\right\rangle, \quad\left|\Psi_{2}\right\rangle=\left(1-i \epsilon R_{2}\right)\left|\Psi_{2}^{G}\right\rangle$,

where $R_{1}, R_{2}$ are generated by $\left\{S_{n}^{(k)}\right\}_{n \leq N}$ for some $N$, meaning that $R_{1}, R_{2}$ do not contain products of $\hat{\phi}$ 's and $\hat{\pi}$ 's that exceed length $N$. The analysis that follows generalizes to arbitrary orders in $\epsilon$.

We will construct a unitary of the form $U=e^{-i(Q+\epsilon R)}$, where $R$ is generated by $\left\{S_{n}^{(k)}\right\}_{n \leq N}$ such that $U\left|\Psi_{1}\right\rangle=$ $\left|\Psi_{2}\right\rangle+\mathcal{O}\left(\epsilon^{2}\right)$. Let $Q$ be a quadratic operator which satisfies $e^{-i Q}\left|\Psi_{1}^{G}\right\rangle=\left|\Psi_{2}^{G}\right\rangle$. Given $\left|\Psi_{1}^{G}\right\rangle,\left|\Psi_{2}^{G}\right\rangle$, we can construct such a $Q$ explicitly. Then, using various manipulations of the Baker-Campbell-Hausdorff formula, we obtain

$$
R=\frac{i \operatorname{ad}_{Q}}{1-e^{i \operatorname{ad}_{Q}}} R_{1}+\frac{i \mathrm{ad}_{Q}}{1-e^{-i \operatorname{ad}_{Q}}} R_{2},
$$

where the superoperator $\operatorname{ad}_{A}$ acts by $\operatorname{ad}_{A} B=[A, B]$. Even though Eq. (5) may appear unwieldy - when we expand out the power series in the $\operatorname{ad}_{Q}$ operators, we find an infinite sum of nested commutators - there is a crucial simplification: the commutator of a quadratic operator with any operator generated by $\left\{S_{n}^{(k)}\right\}_{n \leq N}$ yields another operator which is still in $\left\{S_{n}^{(k)}\right\}_{n \leq N}$. As a consequence, we can write $R$ above as $R=R_{1}^{\prime}+R_{2}^{\prime}$, where $R_{1}^{\prime}, R_{2}^{\prime}$ are each in $\left\{S_{n}^{(k)}\right\}_{n \leq N}$. Furthermore, given specific states $\left|\Psi_{1}\right\rangle,\left|\Psi_{2}\right\rangle$ which are translation and rotation invariant, we can explicitly compute $Q$ as well as $R$ : namely, by explicitly evaluating Eq. (5).

Said in a different way, manipulations of quadratic operators are tractable because $\left\{S_{n}^{(k)}\right\}_{n \leq 2}$ forms a basis for a (closed) Lie algebra. By contrast, nonquadratic operators are harder to handle because $\left\{S_{n}^{(k)}\right\}_{n \leq N}$ for any $N>2$ does not form a basis for a closed Lie algebra, since commutators of nonquadratic operators generically yield operators with progressively longer products of $\hat{\phi}$ 's and $\hat{\pi}$ 's. 
However, $\left\{S_{n}^{(k)}\right\}_{n \leq 2} \cup\left\{\epsilon S_{n}^{(k)}\right\}_{n \leq N}$ does form a basis for a closed Lie algebra to $\mathcal{O}(\epsilon)$, which enables us to evaluate various sums of nested commutators [such as in Eq. (5)] in closed form. At higher orders in $\epsilon$, we would leverage the fact that

$$
\left\{S_{n}^{(k)}\right\}_{n \leq 2} \cup\left\{\epsilon S_{n}^{(k)}\right\}_{n \leq N} \cup \bigcup_{\ell=2}^{m}\left\{\epsilon^{\ell} S_{n}^{(k)}\right\}_{n \leq \ell(N-1)}
$$

forms a basis for a closed Lie algebra to $\mathcal{O}\left(\epsilon^{m}\right)$.

In the next section, we construct a circuit from an arbitrary scale-invariant, zero-mean, Gaussian wave functional to the ground state of $\varphi^{4}$ theory at one loop in the perturbative coupling. In subsequent sections, we will use this result to construct a local position space cMERA which is equivalent to one-loop Wilsonian RG on spatial momentum modes.

\section{ONE-LOOP CIRCUIT FROM GAUSSIAN TO GROUND STATE OF $\varphi^{4}$ THEORY}

It will be convenient for us to work in momentum space. We choose Fourier conventions so that $[\hat{\phi}(\vec{k}), \hat{\pi}(\vec{p})]=$ $i \delta^{d}(\vec{k}+\vec{p})$. The Hamiltonian for $\varphi^{4}$ theory, with one-loop Wilsonian renormalization on its spatial momentum modes, is given by

$$
\begin{aligned}
H_{1-\text { loop }}^{\Lambda e^{u}}= & \frac{1}{2} \int^{\Lambda} d^{d} \vec{k}\left(\hat{\pi}_{\vec{k}} \hat{\pi}_{-\vec{k}}+\hat{\phi}_{\vec{k}}\left(\vec{k}^{2}+e^{-2 u} \tilde{m}^{2}\right) \hat{\phi}_{-\vec{k}}\right) \\
& +\frac{e^{(d-3) u} \lambda}{4 !} \frac{1}{(2 \pi)^{d}} \\
& \times \int^{\Lambda} d^{d} \vec{k}_{1} d^{d} \vec{k}_{2} d^{d} \vec{k}_{3} \hat{\phi}_{\vec{k}_{1}} \hat{\phi}_{\vec{k}_{2}} \hat{\phi}_{\vec{k}_{3}} \hat{\phi}_{-\vec{k}_{1}-\vec{k}_{2}-\vec{k}_{3}},
\end{aligned}
$$

where $\hat{\phi}_{k}:=\hat{\phi}(\vec{k})$. The Hamiltonian is renormalized to scale $|\vec{k}|=\Lambda e^{u}$ with $-\infty<u \leq 0$, and $\tilde{m}^{2}=m^{2}+\delta m^{2}$, with $m$ being the bare mass. The one-loop ground state wave functional of $H_{1-\text { loop }}^{\Lambda e^{u}}$ is [21]

$$
\left\langle\phi \mid \Psi\left(\Lambda e^{u}\right)\right\rangle=\mathcal{N} e^{-G[\phi]-e^{-2 u} \delta m^{2} R_{1}[\phi]-e^{(d-3) u} \lambda R_{2}[\phi]}+\mathcal{O}\left(\lambda^{2}\right),
$$

where $G[\phi], R_{1}[\phi], R_{2}[\phi]$ are specified in the Appendix.

Next we introduce a reference Gaussian state $\left|\Psi_{0}\right\rangle$, namely,

$$
\left\langle\phi \mid \Psi_{0}\right\rangle=\operatorname{det}^{\frac{1}{4}}(\Omega / \pi) e^{-\frac{1}{2} \int d^{d} \vec{k} \phi_{\vec{k}} \Omega(\vec{k}) \phi_{-\vec{k}}},
$$

which is translation and rotation invariant with zero mean.

We construct a unitary $U$ such that $U\left|\Psi_{0}\right\rangle=\left|\Psi\left(\Lambda e^{u}\right)\right\rangle+$ $\mathcal{O}\left(\lambda^{2}\right)$. Our unitary is $U=\exp \left(i K_{2,0}+i \lambda\left(K_{2,1}+K_{4}\right)\right)$ with

$$
\begin{aligned}
K_{2,0} & =-\int d^{d} \vec{k}_{1} d^{d} \vec{k}_{2} \delta^{(d)}\left(\vec{k}_{1}+\vec{k}_{2}\right) g_{2,0}\left(\vec{k}_{1}\right) S_{2}^{(1)}\left(\vec{k}_{1}, \vec{k}_{2}\right) \\
K_{2,1} & =-\int d^{d} \vec{k}_{1} d^{d} \vec{k}_{2} \delta^{(d)}\left(\vec{k}_{1}+\vec{k}_{2}\right) g_{2,1}\left(\vec{k}_{1}\right) S_{2}^{(1)}\left(\vec{k}_{1}, \vec{k}_{2}\right) \\
K_{4}= & \int \prod_{i=1}^{4} d^{d} \vec{k}_{i} \delta^{(d)}\left(\sum_{j=1}^{4} \vec{k}_{j}\right)\left(g_{4}^{(1)}\left(\vec{k}_{1}, \vec{k}_{2}, \vec{k}_{3}, \vec{k}_{4}\right)\right. \\
& \times S_{4}^{(1)}\left(\vec{k}_{1}, \vec{k}_{2}, \vec{k}_{3}, \vec{k}_{4}\right)+g_{4}^{(3)}\left(\vec{k}_{1}, \vec{k}_{2}, \vec{k}_{3}, \vec{k}_{4}\right) \\
& \left.\times S_{4}^{(3)}\left(\vec{k}_{1}, \vec{k}_{2}, \vec{k}_{3}, \vec{k}_{4}\right)\right)
\end{aligned}
$$

where the $g_{2,0}, g_{2,1}, g_{4}^{(1)}$, and $g_{4}^{(3)}$ kernels are specified in the Appendix.

The unitary that we have constructed is not the unique unitary satisfying $U\left|\Psi_{0}\right\rangle=\left|\Psi\left(\Lambda e^{u}\right)\right\rangle+\mathcal{O}\left(\lambda^{2}\right)$. For instance, if we have any unitaries $U_{1}, U_{2}$ satisfying

$$
\begin{aligned}
U_{1}\left|\Psi_{0}\right\rangle & =\left|\Psi_{0}\right\rangle+\mathcal{O}\left(\lambda^{2}\right), \\
U_{2}\left|\Psi\left(\Lambda e^{u}\right)\right\rangle & =\left|\Psi\left(\Lambda e^{u}\right)\right\rangle+\mathcal{O}\left(\lambda^{2}\right),
\end{aligned}
$$

then we have

$$
U_{2} U U_{1}\left|\Psi_{0}\right\rangle=\left|\Psi\left(\Lambda e^{u}\right)\right\rangle+\mathcal{O}\left(\lambda^{2}\right) .
$$

Therefore, $U_{2} U U_{1}$ is also a viable unitary for our purposes. It is in fact possible to construct the most general unitary mapping $\left|\Psi_{0}\right\rangle$ to $\left|\Psi\left(\Lambda e^{u}\right)\right\rangle$ up to $\mathcal{O}\left(\lambda^{2}\right)$ corrections, but we will not do so here. It will suffice to consider our particular unitary $U=\exp \left(i K_{2,0}+i \lambda\left(K_{2,1}+K_{4}\right)\right)$.

\section{CMERA FOR WEAKLY INTERACTING FIELDS}

cMERA is a variational ansatz for the ground states of field theories. The ansatz, which lives in the UV, is constructed by building up entanglement hierarchically from an unentangled, scale-invariant IR state. Concretely, consider the IR state $|\Omega\rangle$ which has the form

$$
\langle\phi \mid \Omega\rangle=\mathcal{N} \exp \left(-\frac{1}{2} \int d^{d} \vec{x} \phi(\vec{x}) M \phi(\vec{x})\right)
$$

for some constant $M$. Notice that $|\Omega\rangle$ is separable (i.e., spatially unentangled) and is scale invariant with respect to spatial dilatations, i.e., $e^{-i u L}|\Omega\rangle=|\Omega\rangle$, with $L$ being the spatial dilatation operator. The cMERA ansatz takes the form of the path-ordered exponential

$$
\left|\Psi_{\mathrm{cMERA}}\right\rangle=\mathcal{P}_{s} \exp \left(-i \int_{u_{\mathrm{IR}}}^{u_{\mathrm{UV}}} d s(K(s)+L)\right)|\Omega\rangle,
$$

where $K(s)$ is called the entangler, which contains free parameters that we variationally optimize by minimizing 
$\left\langle\Psi_{\text {cMERA }}\left|H_{\mathrm{UV}}\right| \Psi_{\text {cMERA }}\right\rangle$ for some UV Hamiltonian. For concreteness, we let $u_{\mathrm{IR}}=-\infty$ and $u_{\mathrm{UV}}=0$. Equation (15) has a straightforward interpretation: $K(s)$ creates correlations at a distance scale $\sim \Lambda^{-1} \exp (-s)$ for $-\infty<s \leq 0$. Or equivalently, in momentum space, $K(s)$ creates correlations at a momentum scale $\sim \Lambda \exp (s)$ for $-\infty<s \leq 0$.

If we want to capture the correlations of $\left|\Psi_{\text {cMERA }}\right\rangle$ in Eq. (15) renormalized down to the momentum scale $\Lambda e^{u}$ (i.e., distance scale $\Lambda^{-1} e^{-u}$ ) for $-\infty \leq u \leq 0$, then we would replace $u_{\mathrm{IR}}$ with $u$.

Even though we have cast cMERA as a variational ansatz, all previously known applications have been for the ground states of free bosonic or free fermionic theories [15-18]. The ground state of a free theory is a Gaussian wave functional, and one can find $K(s)$ exactly so that $\left|\Psi_{\text {cMERA }}(\Lambda)\right\rangle$ agrees with a free ground state in the UV.

One complication with computing $K(s)$ for interacting theories is that their RG flows are nontrivial, unless the theory is a conformal field theory (CFT). Necessarily, $K(s)$ must encode information about the RG flow, so will have a more complicated form vis-à-vis free theories.

In this section, we will use quantum circuit perturbation theory to construct a local $K(s)$ such that the corresponding cMERA state agrees with the one-loop UV ground state of $\varphi^{4}$ theory. Additionally, our cMERA state will have $\left|\Psi_{\text {cMERA }}\left(\Lambda e^{u}\right)\right\rangle$ equal with the one-loop Wilsonian renormalized ground state of $\varphi^{4}$ theory at all intermediate RG scales $\Lambda e^{u}$. This establishes a direct correspondence between cMERA circuits with local entanglers, and Wilsonian RG on spatial momentum modes.

Before proceeding to $\varphi^{4}$ theory, we will first compute $K(s)$ for the ground state of a free massive scalar field theory, such that $\left|\Psi_{\text {CMERA }}\left(\Lambda e^{u}\right)\right\rangle$ equals the Wilsonian renormalized ground state at all intermediate RG scales. This is distinct from previous work, which only required that the cMERA state agree with a desired UV ground state [15-18].

The exact Wilsonian renormalized Hamiltonian for a massive scalar field theory is given by Eq. (7) with $\lambda=0$. The ground state renormalized to scale $\Lambda e^{u}$ is

$$
\left\langle\phi \mid \Psi_{0}\left(\Lambda e^{u}\right)\right\rangle=\mathcal{N} e^{-\frac{1}{2} \int d^{d} \vec{k} \theta(1-|\vec{k}| / \Lambda) \phi_{\vec{k}} \sqrt{\vec{k}^{2}+e^{-2 u} m^{2}} \phi_{-\vec{k}}},
$$

where $\theta(z)$ is an analytic (and thus smooth) version of the Heaviside step function, for instance, a sigmoid. Thus, $\theta(1-|\vec{k}| / \Lambda)$ provides a smooth cutoff at $|\vec{k}|=\Lambda$. (Recall that when we perform Wilsonian RG down to scale $\Lambda e^{u}$, we rescale the momenta so that the cutoff is set back to $\Lambda$, and we also renormalize the fields to put the kinetic term of the Hamiltonian in a canonical form.) Letting $M=\sqrt{\Lambda^{2}+m^{2}}$ in Eq. (14), we find that the desired entangler is

$$
\begin{aligned}
K(s)= & \int d^{d} \vec{k}\left[\frac{1}{4} \theta(1-|\vec{k}| / \Lambda)-\frac{1}{8} \log \left(\frac{\vec{k}^{2}+e^{-2 s} m^{2}}{\Lambda^{2}+m^{2}}\right)\right. \\
& \left.\times \frac{|\vec{k}|}{\Lambda} \theta^{\prime}(1-|\vec{k}| / \Lambda)\right]\left(\phi_{\vec{k}} \pi_{-\vec{k}}+\pi_{\vec{k}} \phi_{-\vec{k}}\right) .
\end{aligned}
$$

Our answer has several interesting features in position space. The Fourier transform of $\frac{1}{4} \theta(1-|\vec{k}| / \Lambda)$ is a function localized at the origin with width $1 / \Lambda$ which leads to correlations at scale $\Lambda e^{s}$ in the cMERA state. The Fourier transform of $-\frac{1}{8} \log \left(\frac{\vec{k}^{2}+e^{-2 s} m^{2}}{\Lambda^{2}+m^{2}}\right) \frac{|\vec{k}|}{\Lambda} \theta^{\prime}(1-|\vec{k}| / \Lambda)$ is localized at the origin with width $1 /\left(e^{-s} m\right)$ (i.e., the inverse renormalized mass scale), but it has essentially zero amplitude unless $1 / m \lesssim \Lambda^{-1} e^{-s}$. This result means that we can only see the effect of the mass $m$ of the UV theory if we probe distance scales around $\sim 1 / \mathrm{m}$ or larger. Probing shorter distance scales essentially only touches massless modes.

Now we construct the entangler $K(s)$ for the ground state of $\varphi^{4}$ theory such that $\left|\Psi_{\text {cMERA }}\left(\Lambda e^{u}\right)\right\rangle$ equals the Wilsonian renormalized ground state at all intermediate $\mathrm{RG}$ scales to one loop. Recall that the desired ground state $\left|\Psi\left(\Lambda e^{u}\right)\right\rangle$ is given by Eq. (8). We find that the one-loop entangler can be written as

$$
\begin{aligned}
K(s)= & \int d^{d} \vec{k}_{1} d^{d} \vec{k}_{2} \delta^{(d)}\left(\vec{k}_{1}+\vec{k}_{2}\right) f_{2,0}\left(\vec{k}_{1} ; s\right) S_{2}^{(1)}\left(\vec{k}_{1}, \vec{k}_{2}\right) \\
& +\lambda \int d^{d} \vec{k}_{1} d^{d} \vec{k}_{2} \delta^{(d)}\left(\vec{k}_{1}+\vec{k}_{2}\right) f_{2,1}\left(\vec{k}_{1} ; s\right) S_{2}^{(1)}\left(\vec{k}_{1}, \vec{k}_{2}\right) \\
& +\lambda e^{d s} \int d^{d} \mathbf{k} \delta^{(d)}\left(\vec{k}_{1}+\vec{k}_{2}+\vec{k}_{3}+\vec{k}_{4}\right) \\
& \times\left(f_{4}^{(1)}\left(\vec{k}_{1}, \vec{k}_{2}, \vec{k}_{3}, \vec{k}_{4} ; s\right) S_{4}^{(1)}\left(\vec{k}_{1}, \vec{k}_{2}, \vec{k}_{3}, \vec{k}_{4}\right)\right. \\
& \left.+f_{4}^{(3)}\left(\vec{k}_{1}, \vec{k}_{2}, \vec{k}_{3}, \vec{k}_{4} ; s\right) S_{4}^{(3)}\left(\vec{k}_{1}, \vec{k}_{2}, \vec{k}_{3}, \vec{k}_{4}\right)\right)
\end{aligned}
$$

where the kernels $f_{2,0}, f_{2,1}, f_{4}^{(1)}, f_{4}^{(3)}$ are given in the Appendix. Although the expressions for the kernels are somewhat unwieldy, they have several remarkable properties. Most importantly, some Fourier analysis shows that all of the kernels in $K(s)$ decay at worst exponentially in position space, with decay constant $\sim 1 /\left(e^{-s} m\right)$ (i.e., the inverse renormalized mass scale). More specifically, the Fourier transform of each kernel decays at worst exponentially in the distance $\left|\vec{x}_{i}-\vec{x}_{j}\right|$ between any two spatial position vectors $\vec{x}_{i}, \vec{x}_{j}$ appearing in the kernel. The locality of the kernels arises due to the poles of the massive propagator, as well as the relativistic dispersion relation for massive fields $\omega_{k}=\sqrt{\vec{k}^{2}+e^{-2 s} m^{2}}$. (Here we have renormalized the mass to momentum scale $\Lambda e^{s}$.) The mass terms shift the poles of the kernels off the real axis (or axes, for the multidimensional integrals), so we can analytically deform the contour of the Fourier transform to achieve exponential decay in position space. We have plotted 

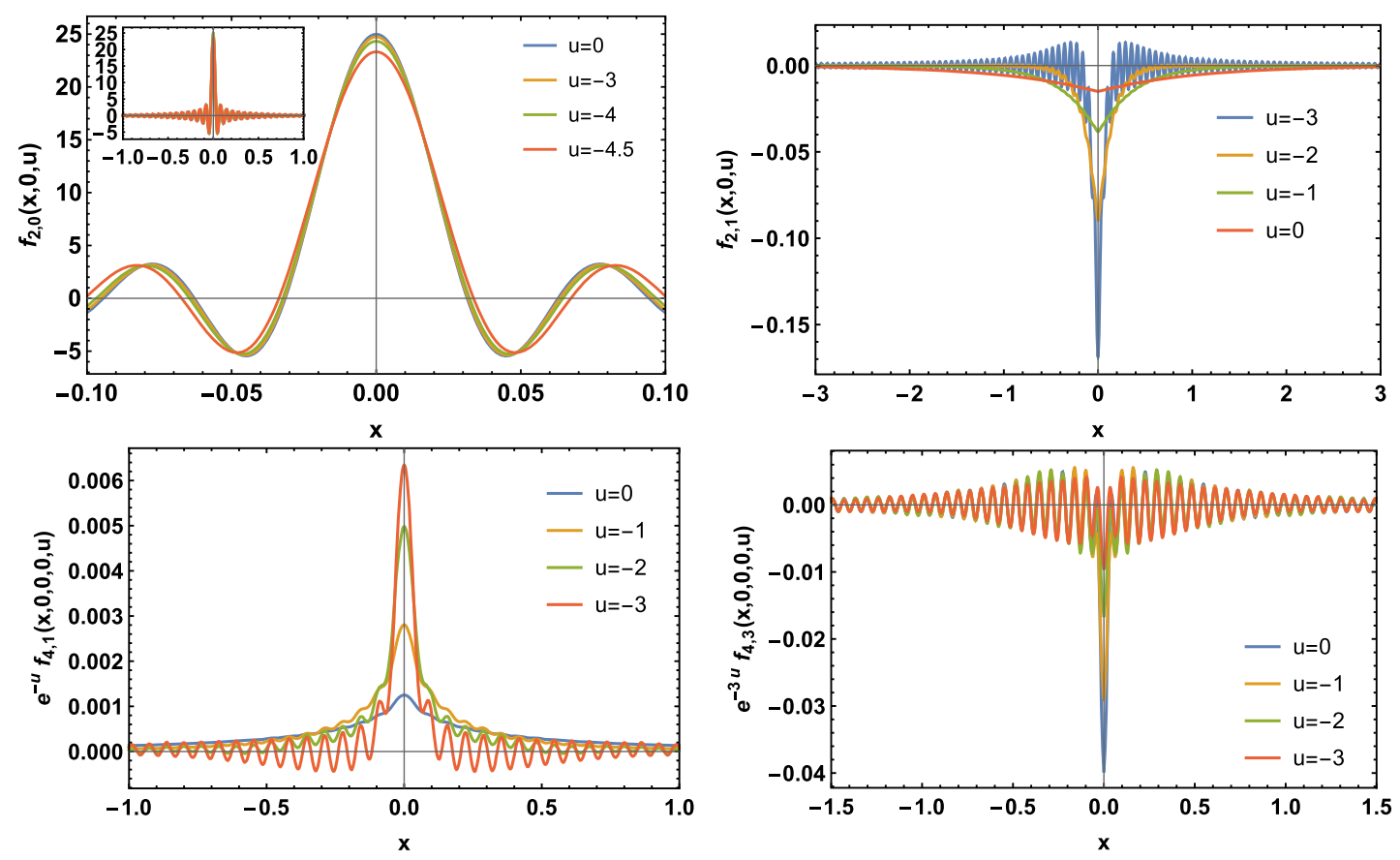

FIG. 1. The kernels comprising the entangler in position space at different values of $u$. Here we have set $m=1$ and $\Lambda=100$.

spatial slices of the kernels in Fig. 1 by taking Fourier transforms into real space.

Our analysis implies that the entangler is exponentially local in position space, and thus for the case at hand, Wilsonian RG on spatial momentum modes can be reexpressed in terms of a local cMERA circuit to one loop in perturbation theory. This creates a direct link between more standard momentum space Wilsonian RG and cMERA tensor networks. Since the exponential position space locality of the kernels only depends on the pole structure of the propagator and the relativistic dispersion relation for massive fields, we expect analogous results to hold to higher loops in $\varphi^{4}$ theory, and for other massive theories. For massless theories, the kernels will have a weaker decay due to the altered pole structure of the propagator and the relativistic dispersion relation for massless fields.

Our result for $K(s)$ for $\varphi^{4}$ theory has the property that the mass-dependent parts of the entangler only "activate" at distance scales greater than $\sim 1 / m$, which is analogous to the free theory result above.

\section{DISCUSSION}

We have shown that we can perturbatively construct a cMERA with a local entangler for the ground state of weakly interacting $\varphi^{4}$ theory. Furthermore, the cMERA can be constructed to agree with Wilsonian RG on spatial momentum modes. Our procedure is systematic and should provide similar constructions for other QFTs. In particular, we expect that cMERA kernels for other massive theories, given by our procedure, will also be exponentially localized in position space. Furthermore, we expect that cMERA kernels for massless theories will also be localized in position space, but not exponentially. Our calculations motivate a numerical approach to cMERA, which does not require fields to be weakly interacting (see the Appendix).

There are several interesting future directions. First, it would be interesting to perform higher-loop calculations, and to generalize the results to fermionic theories [22] and gauge fields. One can also compute the cMERA circuit for weakly interacting CFTs like the Wilson-Fisher fixed point. It may also be possible to generalize our perturbative techniques to many-body spin systems, along the lines of [23].

Since tensor networks are intrinsically tied with entanglement properties of the quantum states they generate, a detailed study of the entanglement properties of weakly interacting cMERA circuits may yield new insights. It would also be interesting to understand the connection to "flow equations" [24-26] and various generalizations of holography [27-30]. One could also explore complexity for weakly interacting field theories, along the lines of $[31,32]$.

\section{ACKNOWLEDGMENTS}

We would like to thank Chris Akers, Ignacio Cirac, William Donnelly, Patrick Hayden, Michal Heller, Javier Molina-Vilaplana, Mark Mueller, Tobias Osborne, Daniel Ranard, Tadashi Takayanagi, Frank Verstraete, and Guifré Vidal for valuable conversations and feedback. We thank Felipe Hernández for many discussions about the locality of the cMERA kernels in position space. J.S.C. is supported by the Fannie and John Hertz Foundation and 
the Stanford Graduate Fellowship program. A.M. acknowledges support by the Alexander von Humboldt Foundation via a postdoctoral fellowship. A. M. also would like to thank ICTP for their hospitality during the last stages of this work. A. N. has greatly profited from discussions with Farhad Ardalan on Wilsonian RG and also Vahid Karimipour and Niloofar Vardian on different aspects of tensor networks. A. N. also would like to thank CERN TH-Division and ICTP for their hospitality during some stages of this work.

\section{APPENDIX}

\section{Kernels for one-loop entangler}

Here we specify some details of our analysis at one loop. The parameters and kernels in the one-loop ground state given in Eq. (8) are

$$
\begin{gathered}
\tilde{m}^{2}=m^{2}+\frac{\lambda}{2} \int_{\Lambda e^{u}}^{\Lambda} \frac{d^{d} \vec{k}}{(2 \pi)^{d}} \frac{1}{\vec{k}^{2}+m^{2}}=: m^{2}+\delta m^{2} \\
G[\phi]=\frac{1}{2} \int^{\Lambda} d^{d} \vec{k} \phi_{\vec{k}} \omega_{k} \phi_{-\vec{k}} \\
R_{1}[\phi]=\frac{1}{4} \int^{\Lambda} d^{d} \vec{k} \frac{1}{\omega_{k}} \phi_{\vec{k}} \phi_{-\vec{k}} \\
R_{2}[\phi]=\frac{1}{16} \int^{\Lambda} d^{d} \vec{k} \frac{1}{\omega_{k}}\left(\int \frac{d^{d} \vec{q}}{(2 \pi)^{d}} \frac{1}{\omega_{k}+\omega_{q}}\right) \phi_{\vec{k}} \phi_{-\vec{k}} \\
+\frac{1}{24} \frac{1}{(2 \pi)^{d}} \int^{\Lambda} \frac{d^{d} \vec{k}_{1} d^{d} \vec{k}_{2} d^{d} \vec{k}_{3}}{\omega_{k_{1}}+\omega_{k_{2}}+\omega_{k_{3}}+\omega_{-\vec{k}_{1}-\vec{k}_{2}-\vec{k}_{3}}} \\
\times \phi_{\vec{k}_{1}} \phi_{\overrightarrow{k_{2}}} \phi_{\vec{k}_{3}} \phi_{-\vec{k}_{1}-\vec{k}_{2}-\vec{k}_{3}},
\end{gathered}
$$

with $\omega_{k}:=\sqrt{\vec{k}^{2}+e^{-2 u} m^{2}}$.

The unspecified kernels $g_{2,0}, g_{2,1}, g_{4}^{(1)}$, and $g_{4}^{(3)}$ in Sec. III are given below. Defining

$$
\begin{aligned}
\mathcal{G}_{j} & :=2\left(g_{2,0}\left(\vec{k}_{1}\right)-g_{2,0}\left(\vec{k}_{4}\right)+(-1)^{\frac{j+1}{2}}\left(g_{2,0}\left(\vec{k}_{2}\right)+g_{2,0}\left(\vec{k}_{3}\right)\right)\right), \\
\tilde{g}_{4}^{(j)} & :=\frac{e^{-\mathcal{G}_{j}}-1}{\mathcal{G}_{j}} g_{4}^{(j)}, \quad j=1,3,
\end{aligned}
$$

the kernels are

$$
g_{2,0}=\frac{1}{4} \log \left(\Omega\left(\vec{k}_{1}\right) / \omega_{k_{1}}\right)
$$

$$
\begin{aligned}
g_{2,1}= & -\frac{1}{\omega_{k_{1}}^{2}}\left(\frac{e^{-2 u}\left(\delta m^{2} / \lambda\right)}{8}\right. \\
& \left.+\frac{e^{(d-3) u}}{32} \frac{1}{(2 \pi)^{d}} \int d^{d} \vec{k}_{2} \frac{1}{\omega_{k_{1}}+\omega_{k_{2}}}\right),
\end{aligned}
$$

$$
\begin{aligned}
& \tilde{g}_{4}^{(1)}=\frac{1}{96} \frac{e^{(d-3) u}}{(2 \pi)^{d}} \frac{1}{\omega_{k_{2}} \omega_{k_{3}} \omega_{k_{4}}\left(\omega_{k_{1}}+\omega_{k_{2}}+\omega_{k_{3}}+\omega_{k_{4}}\right)}, \\
& \tilde{g}_{4}^{(3)}=\frac{1}{32} \frac{e^{(d-3) u}}{(2 \pi)^{d}} \frac{1}{\omega_{k_{4}}\left(\omega_{k_{1}}+\omega_{k_{2}}+\omega_{k_{3}}+\omega_{k_{4}}\right)} .
\end{aligned}
$$

Our desired ground state $\left|\Psi\left(\Lambda e^{u}\right)\right\rangle$ is given by Eq. (8), with the parameters and kernels specified in Eqs. (A1)-(A4). Letting

$$
\begin{aligned}
M= & \sqrt{\Lambda^{2}+m^{2}}+\lambda\left(\frac{1}{2}\left(\delta m^{2} / \lambda\right) \frac{1}{M}\right. \\
& \left.-\frac{4}{(2 \pi)^{d}} \int^{\Lambda} d^{d} \vec{q} \frac{1}{M\left(M+\sqrt{q^{2}+m^{2}}\right)}\right)
\end{aligned}
$$

in Eq. (14), the one-loop entangler is given in Eq. (18), where $f_{2,0}, f_{2,1}$ are

$$
f_{2, i}\left(e^{-u} \vec{k} ; u\right)=\frac{d}{d u}\left[\theta\left(1-|\vec{k}| / \Lambda e^{u}\right) g_{2, i}\left(e^{-u} \vec{k} ; u\right)\right]
$$

for $i=0,1$, and $f_{4}^{(1)}, f_{4}^{(3)}$ are

$$
\begin{aligned}
f_{4}^{(j)}\left(\vec{k}_{1}, \vec{k}_{2}, \vec{k}_{3}, \vec{k}_{4} ; u\right)= & e^{-(d+1) u}\left\{e^{u}\left(d-\frac{\partial \mathcal{F}_{j}(s, u)}{\partial u}\right) \tilde{g}_{4}^{(j)}\left(\vec{k}_{1}, \vec{k}_{2}, \vec{k}_{3}, \vec{k}_{4} ; u\right)-e^{u} \sum_{j=1}^{4} \vec{k}_{j} \cdot \frac{\partial}{\partial \vec{k}_{j}} \tilde{g}_{4}^{(j)}\left(\vec{k}_{1}, \vec{k}_{2}, \vec{k}_{3}, \vec{k}_{4} ; u\right)\right. \\
& \left.+e^{u} \frac{\partial}{\partial u} \tilde{g}_{4}^{(j)}\left(\vec{k}_{1}, \vec{k}_{2}, \vec{k}_{3}, \vec{k}_{4} ; u\right)-\tilde{g}_{4}^{(j)}\left(\vec{k}_{1}, \vec{k}_{2}, \vec{k}_{3}, \vec{k}_{4} ; u\right) \sum_{\ell=1}^{4} \frac{\left|\vec{k}_{\ell}\right|}{\Lambda} \frac{\theta^{\prime}\left(1-\left|\vec{k}_{p}\right| / \Lambda\right)}{\theta\left(1-\left|\vec{k}_{\ell}\right| / \Lambda\right)}\right\} \prod_{p=1}^{4} \theta\left(1-\left|\vec{k}_{p}\right| / \Lambda\right)
\end{aligned}
$$

for $j=1,3$. Here $\mathcal{F}_{1}(s, u)$ and $\mathcal{F}_{3}(s, u)$ are defined by

$$
\mathcal{F}_{j}(s, u):=2 \int_{s}^{u} d t\left(f_{2,0}\left(e^{-t} \vec{k}_{1} ; t\right)-f_{2,0}\left(e^{-t} \vec{k}_{4} ; t\right)+(-1)^{\frac{j+1}{2}}\left(f_{2,0}\left(e^{-t} \vec{k}_{2} ; t\right)+f_{2,0}\left(e^{-t} \vec{k}_{3} ; t\right)\right)\right) .
$$




\section{Lessons for numerics}

The ultimate goal of cMERA is to provide a robust numerical ansatz for the ground state of an interacting quantum field theory. We have focused on developing machinery for perturbative calculations to bring cMERA into the new territory of interacting field theories, albeit weakly interacting. However, we can use insights from our calculations to construct an ansatz which may be viable for numerical variational calculations.

We construct an entangler

$$
\begin{aligned}
K(s)= & \sum_{j_{1}} \int_{1 / \Lambda} d^{d} \vec{x}_{1} f_{j_{1}}\left(\vec{x}_{1} ; u\right) \mathcal{O}_{j_{1}}\left(\vec{x}_{1}\right) \\
& +\sum_{j_{2}} \int_{1 / \Lambda} d^{d} \vec{x}_{1} d^{d} \vec{x}_{2} f_{j_{2}}\left(\vec{x}_{1}, \vec{x}_{2} ; u\right) \mathcal{O}_{j_{2}}\left(\vec{x}_{1}, \vec{x}_{2}\right) \\
& +\cdots+\sum_{j_{n}} \int_{1 / \Lambda} d^{d} \vec{x}_{1} \cdots d^{d} \vec{x}_{n} f_{j_{n}}\left(\vec{x}_{1}, \ldots, \vec{x}_{n} ; u\right) \\
& \times \mathcal{O}_{j_{n}}\left(\vec{x}_{1}, \ldots, \vec{x}_{n}\right)
\end{aligned}
$$

where the position space integrals are cutoff from below at scale $1 / \Lambda$. We take $f_{i_{1}}, \ldots, f_{i_{n}}$ to be functions of a specified form, but with undetermined parameters that we can tune and optimize. We approximate the cMERA circuit $\mathcal{P}_{s} \exp \left(-i \int_{u_{\mathrm{IR}}}^{0} d s(K(s)+L)\right)$ by

$$
\left[e^{-i \Delta u\left(K\left(u_{\mathrm{R}}\right)+L\right)} e^{-i \Delta u\left(K\left(u_{\mathrm{IR}}+\Delta u\right)+L\right)} \cdots e^{-i \Delta u(K(0)+L)}\right]_{T},
$$

where $\Delta u:=-u_{\mathrm{IR}} / N$ for some positive integer $N$, and $[\cdots]_{T}$ denotes that we truncate the terms inside the bracket at order $\mathcal{O}\left((\Delta u)^{T}\right)$. Our cMERA ansatz is

$$
\begin{aligned}
\left|\Psi_{\mathrm{cMERA}}\right\rangle:= & {\left[e^{-i \Delta u\left(K\left(u_{\mathrm{IR}}\right)+L\right)} e^{-i \Delta u\left(K\left(u_{\mathrm{IR}}+\Delta u\right)+L\right)} \ldots\right.} \\
& \left.\times e^{-i \Delta u(K(0)+L)}\right]_{T}|\Omega\rangle,
\end{aligned}
$$

which depends on the functions $f_{i_{1}}, \ldots, f_{i_{n}}$. To utilize this ansatz, we consider a UV Hamiltonian $H_{\mathrm{UV}}$ and perform the numerical minimization

$$
\min _{f_{i_{1}}, \ldots, f_{i_{n}}} \frac{\left\langle\Psi_{\text {cMERA }}\left|H_{\mathrm{UV}}\right| \Psi_{\text {cMERA }}\right\rangle}{\left\langle\Psi_{\text {cMERA }} \mid \Psi_{\text {cMERA }}\right\rangle},
$$

where the denominator is required since $\left|\Psi_{\text {cMERA }}\right\rangle$ is not normalized as given. Our calculations suggest that a good way of parametrizing the $f_{i_{1}}, \ldots, f_{i_{n}}$ is in terms of sineGaussian wavelets, which only depend on the differences of coordinates $\left|\vec{x}_{i}-\vec{x}_{j}\right|$. For instance, we might parametrize a kernel $f\left(\vec{x}_{1}, \vec{x}_{2}\right)$ by

$$
\begin{aligned}
& f\left(\vec{x}_{1}, \vec{x}_{2} ;\left\{a_{j}, b_{j}, c_{j}, d_{j}, \phi_{j}\right\}\right) \\
& \quad=\sum_{j} a_{j} e^{-b_{j}^{2}\left|\vec{x}_{1}-\vec{x}_{2}\right|^{2}+c_{j}\left|\vec{x}_{1}-\vec{x}_{2}\right|} \cos \left(d_{j}\left|\vec{x}_{1}-\vec{x}_{2}\right|+\phi_{j}\right),
\end{aligned}
$$

which is the form of the sum of the real parts of Gabor wavelets. A kernel $f\left(\vec{x}_{1}, \vec{x}_{2}, \vec{x}_{3}, \vec{x}_{4}\right)$ might be parametrized similarly by

$$
\begin{aligned}
& f\left(\vec{x}_{1}, \vec{x}_{2}, \vec{x}_{3}, \vec{x}_{4} ;\left\{a_{j}, \mathbf{B}_{j}, \mathbf{c}_{j}, \mathbf{d}_{j}, \boldsymbol{\phi}_{j}\right\}\right) \\
& \quad=\sum_{j} a_{j} e^{-\mathbf{x}^{T} \mathbf{B}_{j} \mathbf{x}+\mathbf{c}_{j} \cdot \mathbf{x}} \cos \left(\mathbf{d}_{j} \cdot \mathbf{x}+\boldsymbol{\phi}_{j}\right),
\end{aligned}
$$

where $\quad \mathbf{x}:=\left(\left|\vec{x}_{1}-\vec{x}_{2}\right|,\left|\vec{x}_{1}-\vec{x}_{3}\right|,\left|\vec{x}_{1}-\vec{x}_{4}\right|,\left|\vec{x}_{2}-\vec{x}_{3}\right|,\left|\vec{x}_{2}-\vec{x}_{4}\right|\right.$, $\left.\left|\vec{x}_{3}-\vec{x}_{4}\right|\right)$. Here $\mathbf{B}_{j}$ is a $6 \times 6$ matrix of parameters, and $\mathbf{c}_{j}, \mathbf{d}_{j}, \boldsymbol{\phi}_{j}$ are all six-dimensional vectors of parameters. For non-CFTs, the parameters in the two equations above can depend on $u$ and thus have nontrivial dependence on the distance scale.

We envision that by parametrizing the kernels $f_{i_{1}}, \ldots, f_{i_{n}}$ in terms of appropriate sine-Gaussian wavelets, it should be possible for cMERA to become a useful variational method for the ground states of CFTs as well as regular QFTs (for which there are additional parametric dependencies in the kernels). In particular, the integrals and gradient descent procedure required to minimize

$$
\left\langle\Psi_{\text {cMERA }}\left|H_{\text {UV }}\right| \Psi_{\text {cMERA }}\right\rangle /\left\langle\Psi_{\text {cMERA }} \mid \Psi_{\text {cMERA }}\right\rangle
$$

over the parameters of sine-Gaussian wavelets (or similar such wavelets) can be performed efficiently.
[1] S. R. White, Density Matrix Formulation for Quantum Renormalization Groups, Phys. Rev. Lett. 69, 2863 (1992).

[2] U. Schollwock, The density-matrix renormalization group, Rev. Mod. Phys. 77, 259 (2005).

[3] U. Schollwock, The density-matrix renormalization group in the age of matrix product states, Ann. Phys. (Amsterdam) 326, 96 (2011).
[4] G. Vidal, Entanglement Renormalization: An introduction, arXiv:0912.1651.

[5] G. Vidal, Class of Quantum Many-Body States That Can Be Efficiently Simulated, Phys. Rev. Lett. 101, 110501 (2008).

[6] G. Evenbly and G. Vidal, Tensor Network Renormalization, Phys. Rev. Lett. 115, 180405 (2015).

[7] D. Perez-Garcia et al., Matrix product state representations, Quantum Inf. Comput. 7, 401 (2007). 
[8] A. Weichselbaum, F. Verstraete, U. Schollwöck, J. I. Cirac, and J. von Delft, Variational matrix-product-state approach to quantum impurity models, Phys. Rev. B 80, 165117 (2009).

[9] F. Verstraete, V. Murg, and J. I. Cirac, Matrix product states, projected entangled pair states, and variational renormalization group methods for quantum spin systems, Adv. Phys. 57, 143 (2008).

[10] N. Schuch, M. M. Wolf, F. Verstraete, and J. I. Cirac, Computational Complexity of Projected Entangled Pair States, Phys. Rev. Lett. 98, 140506 (2007).

[11] F. Verstraete and J. I. Cirac, Continuous Matrix Product States for Quantum Fields, Phys. Rev. Lett. 104, 190405 (2010).

[12] J. Haegeman, Variational renormalization group methods for extended quantum systems, Ph.D. thesis, Ghent University, 2011.

[13] V. Stojevic, J. Haegeman, I. P. McCulloch, L. Tagliacozzo, and F. Verstraete, Conformal data from finite entanglement scaling, Phys. Rev. B 91, 035120 (2015).

[14] J. Haegeman, J. I. Cirac, T. J. Osborne, H. Verschelde, and F. Verstraete, Applying the Variational Principle to $(1+1)$ Dimensional Quantum Field Theories, Phys. Rev. Lett. 105, 251601 (2010).

[15] J. Haegeman, T. J. Osborne, H. Verschelde, and F. Verstraete, Entanglement Renormalization for Quantum Fields in Real Space, Phys. Rev. Lett. 110, 100402 (2013).

[16] M. Nozaki, S. Ryu, and T. Takayanagi, Holographic geometry of entanglement renormalization in quantum field theories, J. High Energy Phys. 10 (2012) 193.

[17] A. Mollabashi, M. Nozaki, S. Ryu, and T. Takayanagi, Holographic geometry of cMERA for quantum quenches and finite temperature, J. High Energy Phys. 03 (2014) 098.

[18] Q. Hu and G. Vidal, Spacetime Symmetries and Conformal Data in the Continuous Multiscale Entanglement Renormalization Ansatz, Phys. Rev. Lett. 119, 010603 (2017).
[19] J. S. Cotler, J. Molina-Vilaplana, and M. T. Mueller, A Gaussian variational approach to cMERA for interacting fields, arXiv:1612.02427.

[20] J. Cotler et al., Renormalization group circuits for weakly interacting continuum field theories, arXiv:1806.02831.

[21] B. Hatfield, Quantum Field Theory of Point Particles and Strings, Vol. 75 (Westview Press, Boulder, CO, 1998).

[22] J. S. Cotler, M. R. M. Mozaffar, A. Mollabashi, and A. Naseh (to be published).

[23] L. Vanderstraeten, M. Mariën, J. Haegeman, N. Schuch, J. Vidal, and F. Verstraete, Bridging Perturbative Expansions with Tensor Networks, Phys. Rev. Lett. 119, 070401 (2017).

[24] F. Wegner, Flow-equations for Hamiltonians, Ann. Phys. (N.Y.) 506, 77 (1994).

[25] S. Kehrein, The Flow Equation Approach to Many-Particle Systems (Springer, Berlin, 2006).

[26] S. Kehrein, Flow equation holography, arXiv:1703.03925.

[27] M. Miyaji and T. Takayanagi, Surface/state correspondence as a generalized holography, Prog. Theor. Exp. Phys. 2015, 073 B03 (2015).

[28] M. Miyaji, T. Numasawa, N. Shiba, T. Takayanagi, and K. Watanabe, Continuous Multiscale Entanglement Renormalization Ansatz as Holographic Surface-State Correspondence, Phys. Rev. Lett. 115, 171602 (2015).

[29] M. Miyaji, T. Takayanagi, and K. Watanabe, From path integrals to tensor networks for the AdS/CFT correspondence, Phys. Rev. D 95, 066004 (2017).

[30] P. Caputa, N. Kundu, M. Miyaji, T. Takayanagi, and K. Watanabe, Anti-de Sitter Space from Optimization of Path Integrals in Conformal Field Theories, Phys. Rev. Lett. 119, 071602 (2017).

[31] R. Jefferson and R. C. Myers, Circuit complexity in quantum field theory, J. High Energy Phys. 10 (2017) 107.

[32] S. Chapman, M. P. Heller, H. Marrochio, and F. Pastawski, Toward a Definition of Complexity for Quantum Field Theory States, Phys. Rev. Lett. 120, 121602 (2018). 